\title{
Biofeedback therapy in the treatment of pelvic floor outlet obstruction
}

\author{
DN Samarasekera ${ }^{1}$ CTM Speakman ${ }^{2}$

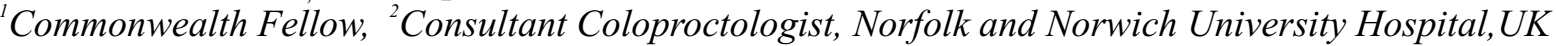

\begin{abstract}
Introduction: Biofeedback treatment in coloproctology refers to a non-invasive method of cognitively retraining the anal sphincter and the pelvic floor muscles in which the patients are guided by the electromyographic (EMG) activity or the pressure (strength of contraction) of the contracting muscles. In coloproctology, biofeedback therapy has been tried both in the management of chronic constipation due to pelvic floor outlet obstruction (PFOO) and faecal incontinence, with mixed results.
\end{abstract}

Materials and methods: From June 1999 up to October 2004, 29 female patients underwent biofeedback therapy for PFOO using an anal pressure sensor probe. The results of biofeedback were graded according to the degree of improvement of symptoms ranging from cure to no improvement (i.e. cure, mild, moderate or significant improvement or no improvement). Repeat biofeedback and anal surface EMG were carried out to confirm improvement or failure.

Results: A total of 29 female patients underwent biofeedback therapy. Mean age was 25.5 years (range, 19-36 years). Eleven patients (37.9\%) did not complete the course. Of the 18 patients who completed the course, all reported varying degrees of relief indicating an overall success rate of $62.1 \%$. This included significant improvement of symptoms in 8 (27.6\%), moderate improvement in $8(27.6 \%)$, and a cure in $2(6.9 \%)$ patients.

Conclusions: Biofeedback therapy gives successful outcome in the majority of patients suffering from PFOO, but needs motivation and strict follow-up to prevent non-compliance.

\section{Introduction}

The term biofeedback describes a therapeutic technique where a subject learns to modify or control a physiological function of the body. The basis of biofeedback is learning through reinforcement. This type of learning is also sometimes called instrumental learning or operant conditioning [1].

With the invention of new diagnostic tests, more and more pathophysiological disorders of the pelvic floor are now being recognised. Pelvic floor outlet obstruction (PFOO) is a rare cause of chronic constipation where during defaecation the patient contracts the puborectalis muscle (instead of relaxing) leading to an increase in the anorectal angle, thereby preventing rectal evacuation (obstructive constipation). In chronic constipation due to PFOO (also known as anismus, pelvic floor dyssynergia, spastic pelvic floor syndrome, paradoxical puborectalis contraction and puborectalis syndrome) biofeedback is carried out to teach the patient to relax (instead of contracting) the external anal sphincter and the puborectalis during defaecation [2].

\section{Materials and methods}

From June 1999 up to October 2004, 29 female patients underwent biofeedback therapy for pelvic floor outlet obstruction (PFOO) using an anal pressure sensor probe (Figure-1). The diagnosis of PFOO in all the patients was confirmed by anal surface electromyography (EMG) and defaecation proctography (video floroscopy of a defaecating barium enema). 


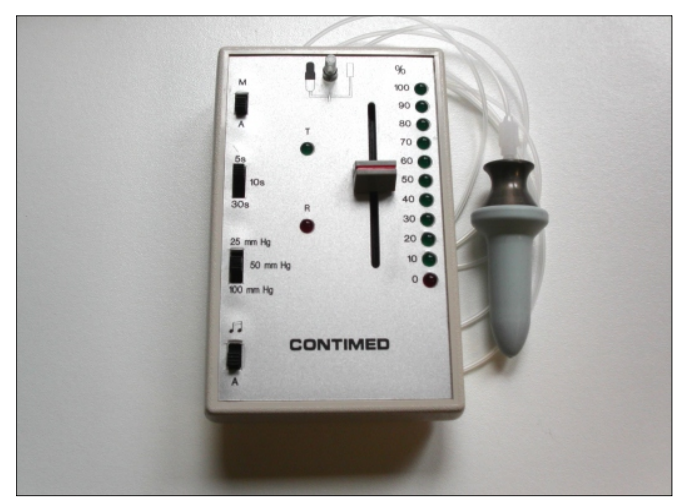

Figure 1 - Contimed biofeedback device with anal pressure sensor

All the patients were also subjected to a psychological evaluation to exclude depression and behavioural disorders. Anorectal physiology studies and volumetry were carried out to exclude Hirschsprung's disease and sphincter abnormalities that may give rise to similar symptoms. Colon transit studies and the endocrine profile were performed to exclude transit abnormalities. Barium enema was carried out to exclude megacolon/rectum and rectocele that may mimic PFOO.

The results of biofeedback were graded according to the degree of improvement of symptoms ranging from, cure to no improvement (i.e. cure, mild, moderate or significant improvement or no improvement). Once the biofeedback therapy course was completed, each patient was examined in the physiology laboratory with a repeat biofeedback (by the clinical physiologist) and anal surface EMG to comfirm improvement or failiure.

Each patient was requested to commit themselves to an eight week period of therapy and to use the biofeedback device (Contimed Device, Neen Healthcare, Dereham, Norfolk, UK - (Figure-1), preferably every day. They were requested to attend the hospital for approximately one hour at the start of their biofeedback course. Once they attended their first session, they were briefed about the device and were shown how to operate it properly. The patients were also given an instruction manual and were taken through the manual with particular emphasis on the need to proceed at a pace appropriate for them. Once the patient had learnt the correct procedure the patient was sent home with instructions on how to contact the hospital if necessary.

\section{Results}

Eleven patients $(37.9 \%)$ did not complete the course. Mean age was 25.5 years (range, 1936years). Biofeedback treatment was considered successful when the patient was able to reduce the electrical activity of the pelvic floor during straining to defaecate. Of the 18 patients who completed the course, all reported varying degrees of relief indicating an overall success rate of $62.1 \%$. This included significant improvement of symptoms in 8 (27.6\%), moderate improvement in $8(27.6 \%)$, and a cure in $2(6.9 \%)$ patients.

\section{Discussion}

Biofeedback treatment in coloproctology refers to a non-invasive method of cognitively retraining the anal sphincter and the pelvic floor muscles in which the patients are guided by the electromyographic (EMG) activity or the pressure (strength of contraction) of the contracting muscles. In 1967, Haskell and Rovner demonstrated that patients could utilise the auditory display of needle EMG activity of the external anal sphincter during clinical investigation to improve control over external anal sphincter function [3]. However, due to the discomfort of this procedure, this technique did not gain popularity until it was replaced by transcutaneous EMG recording, first reported by Macleod in 1979. First documented case of biofeedback training of pelvic floor was a case report by Kohlenberg in 1973 [4]. He reported a successful outcome of biofeedback therapy in a case of a 13 year old boy who developed faecal incontinence following surgery for Hirschsprung's disease. Using a $3 \mathrm{~cm}$ balloon he taught the subject to increase the anal pressure, and reported an increase in the resting anal pressure. 
In coloproctology, biofeedback therapy has been tried both in the management of faecal incontinence and chronic constipation due to PFOO with mixed results [5]. The patients in our study were instructed to synchronise voluntary contractions and relaxations with electrically applied stimuli to the muscle and to perform home exercises. In the past, these exercises were carried out as regular clinic sessions under the supervision of a clinical physiologist but now home exercise programmes have been devised and the patients can be initially shown how to carryout the procedure correctly at the clinic and subsequent clinic visits are only used to monitor the progress of therapy. This has greatly helped to cut down on hospital costs but needs more patient compliance to achieve success as non-compliance is a major drawback, as seen in our study $(37.9 \%)$. Therefore, unless the patients are well motivated home exercise programmes are likely to fail.

A substantial number of these patients with PFOO will be on expensive long-term laxatives and will ultimately be re-referred for further investigations resulting in major expenditure to the health system. Therefore, biofeedback may be the treatment of choice for this rare group of patients as all other treatment procedures such as surgery (i.e. anorectal myectomy, puborectalis muscle incision) and even injection of botulinum toxin have been found to be ineffective [6-8] .

Our biofeedback programme has not shown adequate patient compliance, as over one third $(37.9 \%)$ of the recruited patients defaulted. The possible reason may be the reluctance by the patients to carryout biofeedback as a home based treatment programme, either due to lack of privacy or due to heavy occupational commitments. Therefore, if a successful biofeedback programme is to be implemented, despite the costs involved, a hospital based treatment programme may be the only option.

\section{Conclusion}

Therefore, in conclusion, we wish to state that biofeedback therapy gives successful outcome in the majority of patients suffering PFOO. However, to obtain optimum results of biofeedback, it should be carried out on well motivated patients and in addition, needs a strict follow-up protocol to prevent non-compliance.

\section{References}

1. Engel BT, P Nikoomanesh, and MM Schuster. Operant conditioning of rectosphincteric responses in the treatment of fecal incontinence. New Engalnd Journal of Medicine, 1974; 290(12): 646-9.

2. Duthie GS and DC Bartolo. Anismus: the cause of constipation? Results of investigation and treatment. World Journal of Surgery, 1992; 16(5): 831-5.

3. Haskell B, H Rovner. Electromyography in the management of the incompetent anal sphincter. Disease Colon Rectum, 1967: 10(2): 81-4.

4. Kohlenberg RJ. Operant conditioning of human anal sphincter pressure. Journal of Applied Behavior Analysis, 1973; 6: 201-8.

5. Ko CY, et al, Biofeedback is effective therapy for fecal incontinence and constipation. Archive Surgery, 1997; 132(8): 829-34.

6. Barnes PR, et al, Experience of posterior division of the puborectalis muscle in the management of chronic constipation. British Journal of Surgery, 1985; 72(6): 475-7.

7. Kamm MA, PR Hawley, and JE Lennard-Jones. Lateral division of the puborectalis muscle in the management of severe constipation. British Journal of Surgery, 1988; 75(7): 661-3.

8. Hallan RI, et al, Treatment of anismus in intractable constipation with botulinum A toxin. The Lancet, 1988;2(8613): 714-7. 